\title{
BMJ Open Anti-diabetic agents for prevention of type 2 diabetes mellitus in people with pre-diabetes: a systematic review and network meta-analysis protocol
}

\author{
Xianzhe Wang, ${ }^{1}$ Jiabin Liu, ${ }^{1}$ Lijin Huang, ${ }^{1}$ Hai Zeng, ${ }^{1}$ Guoxin $\mathrm{He},{ }^{2}$ Ling Chen, ${ }^{3}$ \\ Rui Ma, Wenbin Fu, ${ }^{3,4}$ Baile Ning ${ }^{3,4}$
}

To cite: Wang X, Liu J, Huang L, et al. Anti-diabetic agents for prevention of type 2 diabetes mellitus in people with pre-diabetes: a systematic review and network metaanalysis protocol. BMJ Open 2019;9:e029073. doi:10.1136/ bmjopen-2019-029073

- Prepublication history and additional material for this paper are available online. To view these files, please visit the journal online (http://dx.doi. org/10.1136/bmjopen-2019029073).

Received 10 January 2019 Revised 23 July 2019 Accepted 23 July 2019

Check for updates

(C) Author(s) (or their employer(s)) 2019. Re-use permitted under CC BY-NC. No commercial re-use. See rights and permissions. Published by BMJ.

For numbered affiliations see end of article.

Correspondence to

Baile Ning;

HDNJSWLS@yeah.net

Wenbin Fu; zjkzh@139.com

Ling Chen;

HDNJSWLS1@126.com

Rui Ma;

13751714371@163.com

\section{ABSTRACT}

Introduction Type 2 diabetes mellitus (T2DM) is a substantial health problem worldwide. Pre-diabetic state is associated with increased risk for the development of diabetes. There are various pharmacological therapies with glucose-lowering activity for diabetes prevention. Of those, most are being compared with placebo instead of active agents. The relative effects and safety of different glucose-lowering drugs still remain uncertain. To address this gap, we will conduct a systematic review and network meta-analysis (NMA) to evaluate comparative efficacy and safety of glucose-lowering agents for T2DM prevention in patients with pre-diabetes.

Methods and analysis PubMed, the Cochrane library and Embase will be searched from inception to December 2019 for relevant randomised controlled trials (RCTs) that examined anti-diabetic drugs for diabetes prevention in patients with pre-diabetes. Two reviewers working independently will screen titles, abstracts and full papers. Data extraction will also be completed by two independent authors. The primary outcome will be the incidence of T2DM in patients with pre-diabetes at baseline. Secondary outcomes will include the achievement of normoglycaemia, all-cause mortality, cardiovascular mortality and hypoglycaemic event. Pairwise meta-analysis and NMA will be conducted for each outcome using a frequentist random-effects model. Additionally, subgroup analyses will also be performed. The comparison-adjusted funnel plot will be used to assess publication bias. The overall quality of evidence will be rated with the Grading of Recommendations Assessment, Development and Evaluation framework. Data analysis will be conducted using Stata V.14.0.

Ethics and dissemination Ethics approval is not required. We plan to submit the results of this study to a peer-review journal.

PROSPERO registration number CRD42019119157.

\section{INTRODUCTION}

Type 2 diabetes mellitus (T2DM) is a chronic and complex disease, related to insulin secretory defects frequently on the background of insulin resistance; the progression of the disease is associated with genetic factors, metabolic stress and inflammation. ${ }^{1}$ The
Strengths and limitations of this study

- This is a comprehensive systematic review and network meta-analysis (NMA) to evaluate the effectiveness and safety of various glucose-lowering medications on diabetes prevention among people with pre-diabetic state.

- Where possible, an NMA will combine direct evidence with indirect evidence, allowing comparisons of treatments without being compared to each other head-to-head in clinical trials.

- This research will generate clinically useful evidence to benefit patients, clinicians and guideline-makers.

- The different frequencies, dosages and routes of administration of pharmacological therapies may lead to considerable heterogeneity.

global prevalence of T2DM was estimated to be 463 million people in $2017 .^{2}$ People with T2DM are at elevated risk for chronic kidney disease, heart failure, atherosclerotic cardiovascular disease, polyneuropathy, cognitive impairment, anxiety disorder and depression. ${ }^{3-5}$ The term pre-diabetes is used to describe a blood glucose level higher than the normal range but below the cut-off value for T2DM. ${ }^{6}$ Different glycaemic measurements to define the pre-diabetic stage exist, including impaired fasting glucose (IFG), impaired glucose tolerance (IGT) and elevated glycosylated haemoglobin A1c. ${ }^{1}$ The International Diabetes Federation estimated that, in 2017, approximately 352 million persons globally had IGT, which is projected to exceed half a billion people before 2045 . $^{7}$ Hyperglycaemia is a well-described risk factor for all-cause mortality, total number of all-age deaths attributable to high fasting plasma was 6.5 million people in $2017,{ }^{8}$ with T2DM accounting for 1 million deaths. ${ }^{9}$ Moreover, the economic burden of diabetes is large; in 2017, the American Diabetes 
Association (ADA) estimated the total economic costs attributable to diabetes in the USA to be $\$ 327$ billion. ${ }^{1011}$ Thus, there is an urgent need to address huge burden of this worldwide disease with a growing number of suffers. Early interventions for preventing T2DM are warranted. ${ }^{10}$ Persons diagnosed with pre-diabetes are thought to be at increased risk for developing T2DM, the estimated incidence rate of diabetes among patients with pre-diabetes in the following 10 years exceeds one-third. ${ }^{12}$ These people are ideal candidates for diabetes prevention efforts.

To prevent the progression of pre-diabetes to T2DM, an intensive behavioural lifestyle intervention programme (eg, medical nutrition therapy and physical activity) is recommended in the ADA guidelines. ${ }^{13}$ Besides lifestyle modification, a variety of anti-diabetic agents (eg, glucagon-likepeptide (GLP)-1 analogues, metformin and thiazolidinediones) have been investigated in clinical trials for diabetes prevention. These pharmacological approaches with intrinsic glucose-lowering activity (eg, improve the insulin resistance and preserve pancreatic $\beta$-cell function) are recommended for glycaemic treatment in patients with T2DM. ${ }^{14}$ Of these medications, only metformin therapy for diabetes prevention is recommended as an option for patients with pre-diabetes. ${ }^{13}$ However, to date, whether other glucose-lowering agents should be considered in those patients or not has not yet to be clarified clearly, even though some findings of recent studies have demonstrated that these pharmacological agents could also exert benefits to prevent or delay the progression to T2DM. In addition, head-to-head comparisons of different anti-diabetic agents have rarely been performed by previous clinical trials. A network meta-analysis (NMA) method is able to combine direct and indirect evidence and assess comparative efficacy and safety of various interventions. ${ }^{15-17}$ Therefore, we plan to conduct the systematic review and NMA to assess comparative effects and safety of various anti-diabetic medications in preventing T2DM in patients with pre-diabetes.

\section{METHODS}

\section{Study design and registration}

This systematic review protocol is reported in line with the Preferred Reporting Items for Systematic Review and Meta-Analysis Protocols (PRISMA-P) guidelines. ${ }^{18} 19$ This study will be performed in accordance with the PRISMA extension statements for NMA. ${ }^{15}$

\section{Eligibility criteria}

Population

Adults (older than 18 years) who have pre-diabetes will be eligible for inclusion. In this study, pre-diabetic state involves separate IFG, separate IGT or both. Diagnostic criteria for pre-diabetes should be established and described in eligible trials.

\section{Intervention and comparator}

This study will investigate comparisons of anti-diabetic drugs versus another anti-diabetic agent, lifestyle interventions (diet, exercise or both), placebo or no intervention. Anti-diabetic agents include alpha-glucosidase inhibitors (eg, acarbose and voglibose), sulphonylureas (eg, glipizide and glimepiride), meglitinide analogues (eg, nateglinide), dipeptidyl-peptidase (DPP)-4 inhibitors (eg, linagliptin and vildagliptin), GLP-1 analogues (eg, exenatide and liraglutide), biguanides (eg, metformin), thiazolidinediones (eg, rosiglitazone and pioglitazone), alone or in combination. In addition, studies using vitamins, traditional Chinese medicines and alternative therapies will be excluded.

\section{Outcomes}

The primary outcome will be the incidence of T2DM in patients with pre-diabetes at baseline. Secondary outcomes will include the achievement of normoglycaemia, all-cause mortality, cardiovascular mortality and hypoglycaemic event. Classification and definition of T2DM could be based on any recognised standard diagnosis criteria (eg, the ADA guidelines).

\section{Type of studies}

All randomised controlled trials (RCTs) comparing anti-diabetic drugs with another anti-diabetic agent, lifestyle interventions, placebo or no intervention for T2DM prevention in patients with pre-diabetes will be included in this study. Duration of intervention has to be with a minimum of 12 weeks.

\section{Search strategy}

Several databases will be searched from inception to December 2019 for RCTs that investigated anti-diabetic agents for prevention of diabetes among patients with pre-diabetes. The databases will include PubMed, Embase and the Cochrane Library. In addition, the language of publication will be limited to English. Any potentially-relevant article will be retrieved for review. Details of search strategy of PubMed database are shown in the supplemental material. The literature search will be conducted using the following keywords: alpha-glucosidase inhibitors, sulphonylureas, glinides, DPP-4 inhibitors, GLP-1 analogues, biguanides, thiazolidinediones, diabetes, T2DM, pre-diabetes, pre-diabetic state, glucose intolerance, impaired glucose, conversion, delay and prevent. Moreover, all drug names in each drug class will be included in key search terms, for instance, acarbose, voglibose, metformin, glipizide, glimepiride, linagliptin, vildagliptin, nateglinide, liraglutide, exenatide, rosiglitazone and pioglitazone. To identify other eligible studies, reference lists of relevant publications (including trials, reviews and meta-analyses) will be reviewed for a manual search.

\section{Selection of studies}

In accordance with the prespecified inclusion criteria, two reviewers working independently will evaluate all 
titles and abstracts to eliminate papers that were deemed irrelevant. The remaining articles will be included in the further assessment. Reviewers will scrutinise full text for each potentially-relevant article. The study identification and exclusion process will be depicted using the PRISMA flow diagram. Discrepancies in study selection will be resolved by negotiation.

\section{Data collection process}

Two independent reviewers will use a standardised data form to extract trial information. All disagreements will be settled via discussion with the third reviewer. The data extracted will be as follows:

- Patient characteristics (age, gender, race and glycaemic parameters).

- Trial characteristics (author, year of publication, study design, number of participants, country setting and funding information).

- Details of intervention and control (dosage, frequency and treatment duration).

- Data on the outcomes mentioned above.

\section{Assessment of methodological quality}

The Cochrane risk of bias assessment tool will be used to assess risk of bias for individual studies. This method includes the following seven domains: random sequence generation, allocation concealment, blinding of participants and personnel, blinding of outcome assessment, incomplete outcome data, selective reporting and other bias. ${ }^{20}$ Each item will be classified into one of three categories as follows: unclear, high or low risk. All discrepancies in quality assessment will be resolved after mutual agreement and discussion.

\section{Data synthesis and statistical analysis}

Initially, we will use a random-effects approach to pool effect estimates for all treatment comparisons in conventional pairwise meta-analyses. For categorical outcomes, the pooled estimates as risk ratios (RRs) with 95\% CIs will be reported. When data is available, to observe whether the effects of medications on diabetes prevention remain after intervention withdrawn, the pooled RRs for diabetes of the intervention and wash-out or follow-up periods, respectively, will be estimated. Continuous data will be reported as mean differences with their respective $95 \%$ CIs. Statistical heterogeneity across trials will be examined using the $I^{2}$ statistic. The $I^{2}$ statistic of $75 \%, 50 \%$ or $25 \%$ indicates high, moderate or low heterogeneity, separately. ${ }^{21}$ Then, an NMA will be conducted with a frequentist random-effects model. Local inconsistency between direct and indirect evidence within each closed loop will be assessed using a node-splitting test. ${ }^{22}{ }^{23}$ In addition, a 'design-by-treatment' model will be applied to evaluate the assumption of consistency in the whole network. ${ }^{22} \mathrm{We}$ will generate the surface under the cumulative ranking curve (SUCRA) to assess probabilities of interventions in superiority regarding efficacy and safety outcomes, with higher SUCRA values indicating better effects or safety. ${ }^{24}$
The level of significance will be set at an alpha of 0.05. All analyses will be performed with Stata V.14.0.

\section{Subgroup analyses}

Where possible, analyses will be stratified by age (18-45 years and at least 45 years), gender, ethnicity and BMI $\left(25-29.9 \mathrm{~kg} / \mathrm{m}^{2}\right.$ and $\left.\geq 30 \mathrm{~kg} / \mathrm{m}^{2}\right)$. Moreover, we will also perform subgroup analyses according to diagnostic criteria of pre-diabetes (IFG and IGT).

\section{Publication bias}

We will use the comparison-adjusted funnel plot to assess small study effects including publication bias at the network level. ${ }^{25}$

\section{Quality of evidence}

The quality of evidence of estimates derived from this study will be rated using the Grading of Recommendations Assessment, Development and Evaluation (GRADE framework. The GRADE approach characterises the quality of evidence according to publication bias, study limitations, inconsistency, imprecision, and indirectness. ${ }^{26}$ Evidence of efficacy outcomes will be rated from high quality to very low quality.

\section{Patient and public involvement}

No patients or public will participate in the study.

\section{Ethics and dissemination}

Since confidential patient data will not be involved in this study, formal ethics approval is not required. The framework of the PRISMA statements for NMA will be applied to guide review authors to perform this study. The results will be disseminated by a peer-reviewed publication.

\section{DISCUSSION}

This study is a comprehensive systematic review and NMA to compare a variety of anti-diabetic agents for preventing the development of T2DM in patients with pre-diabetes. Our study will provide a summary of available evidence concerning various anti-hyperglycaemia agents for T2DM prevention in patients with pre-diabetic state, benefiting for clinicians and guideline-makers. Previous relevant reviews and meta-analyses ${ }^{27-29}$ only included clinical trials published before 2015. Importantly, recent large-scale RCTs (eg, the ACE and IRIS trials) ${ }^{30} 31$ have provided substantial data with respect to this topic. Additionally, DPP-4 inhibitors and glucagon-like peptide (GLP)-1 analogues are not involved in previous studies. It is essential to contain these commonly prescribed medications in multiple comparisons of glucose-lowering agents for the prevention of T2DM. Moreover, the influence of different diagnostic criteria for pre-diabetes (IFG and IGT) on the prevention efficacy of anti-diabetic agents remains uncertain. ${ }^{28}$ Thus, we plan to conduct this study to investigate various anti-diabetic agents for diabetes prevention. The findings of our study will generate high quality recommendations regarding the optimal anti-diabetic agent to 
reduce risk of diabetes for patients with pre-diabetes. This study will combine data of all glucose-lowering drugs that have been tested for diabetes prevention by clinical trials. To develop better individualised strategies for diabetes prevention, intervention efficacy according to diagnostic criteria for pre-diabetes (IFG and IGT) will also be explored. However, our study may have several possible limitations. First, the different frequencies, dosages, and routes of administration of pharmacological therapies may result in considerable heterogeneity. Second, differences in the inclusion criteria of participants and definition of the primary end-point events may influence the quality of evidence. Finally, study level data will be used rather than data on individuals.

\section{Author affiliations}

${ }^{1}$ The Second Clinical College of Guangzhou University of Chinese Medicine, Guangzhou, China

${ }^{2}$ The First College of Guangzhou University of Chinese Medicine, Guangzhou, China ${ }^{3}$ Department of Acupuncture and Moxibustion, Guangdong Provincial Hospital of Chinese Medicine, Guangzhou, China

${ }^{4}$ Shenzhen Bao'an Research Center for Acupuncture and Moxibustion, Shenzhen, China

Contributors $\mathrm{HZ}$ and $\mathrm{XW}$ conceived the review. BN, GH, JL, and XW wrote the first draft of this protocol. WF, LC, LH, and RM were responsible for revision of the draft. $\mathrm{HZ}$, LH, and $\mathrm{JL}$ contributed to developing the search strategy and registering the protocol. WF and $\mathrm{HZ}$ were the guarantors. All authors scrutinized and approved the final manuscript.

Funding This research was supported by Guangzhou University of Chinese Medicine, Guangdong Provincial Hospital of Chinese Medicine (No. E43603 and E43703), and the Sanming Project of Medicine in Shenzhen (No. SZSM201806077).

Competing interests None declared.

Patient consent for publication Not required.

Provenance and peer review Not commissioned; externally peer reviewed.

Open access This is an open access article distributed in accordance with the Creative Commons Attribution Non Commercial (CC BY-NC 4.0) license, which permits others to distribute, remix, adapt, build upon this work non-commercially, and license their derivative works on different terms, provided the original work is properly cited, appropriate credit is given, any changes made indicated, and the use is non-commercial. See: http://creativecommons.org/licenses/by-nc/4.0/.

\section{REFERENCES}

1. American Diabetes Association. 2. Classification and Diagnosis of Diabetes: Standards of Medical Care in Diabetes-2019. Diabetes Care 2019;42(Suppl 1):S13-28.

2. GBD 2017 Disease and Injury Incidence and Prevalence Collaborators. Global, regional, and national incidence, prevalence, and years lived with disability for 354 diseases and injuries for 195 countries and territories, 1990-2017: a systematic analysis for the global burden of disease study 2017. Lancet 2018;392:1789-858.

3. American Diabetes Association. 10. Cardiovascular Disease and Risk Management: Standards of Medical Care in Diabetes-2019. Diabetes Care 2019;42(Suppl 1):S103-23.

4. American Diabetes Association. 11. Microvascular Complications and Foot Care: Standards of Medical Care in Diabetes-2019. Diabetes Care 2019;42(Suppl 1):S124-38.

5. Callaghan BC, Xia R, Reynolds E, et al. Association between metabolic syndrome components and polyneuropathy in an obese population. JAMA Neurol 2016;73:1468-76.

6. Moelands SV, Lucassen PL, Akkermans RP, et al. Alpha-glucosidase inhibitors for prevention or delay of type 2 diabetes mellitus and its associated complications in people at increased risk of developing type 2 diabetes mellitus. Cochrane Database Syst Rev 2018;12.
7. International Diabetes Federation. IDF diabetes atlas. 8th edn. Brussels, Belgium: International Diabetes Federation, 2017.

8. GBD 2017 Risk Factor Collaborators. Global, regional, and national comparative risk assessment of 84 behavioural, environmental and occupational, and metabolic risks or clusters of risks for 195 countries and territories, 1990-2017: a systematic analysis for the global burden of disease study 2017. Lancet 2018;392:1923-94.

9. GBD 2017 Causes of Death Collaborators. Global, regional, and national age-sex-specific mortality for 282 causes of death in 195 countries and territories, 1980-2017: a systematic analysis for the global burden of disease study 2017. Lancet 2018;392:1736-88.

10. Shrestha SS, Honeycutt AA, Yang W, et al. Economic costs attributable to diabetes in each U.S. state. Diabetes Care 2018;41:2526-34.

11. American Diabetes Association. Economic costs of diabetes in the U.S. in 2017. Diabetes Care 2018;41:917-28.

12. Morris DH, Khunti K, Achana F, et al. Progression rates from HbA1c 6.0-6.4\% and other prediabetes definitions to type 2 diabetes: a meta-analysis. Diabetologia 2013;56:1489-93.

13. American Diabetes Association. 3. Prevention or Delay of Type 2 Diabetes: Standards of Medical Care in Diabetes-2019. Diabetes Care 2019;42(Suppl 1):S29-33.

14. American Diabetes Association. 9. Pharmacologic Approaches to Glycemic Treatment: Standards of Medical Care in Diabetes-2019. Diabetes Care 2019;42(Suppl 1):S90-102.

15. Hutton B, Salanti G, Caldwell DM, et al. The PRISMA extension statement for reporting of systematic reviews incorporating network meta-analyses of health care interventions: checklist and explanations. Ann Intern Med 2015;162:777-84.

16. Rouse B, Cipriani A, Shi Q, et al. Network meta-analysis for clinical practice guidelines: a case study on first-line medical therapies for primary open-angle glaucoma. Ann Intern Med 2016;164:674-82.

17. Mills EJ, Thorlund K, loannidis JPA. Demystifying trial networks and network meta-analysis. BMJ 2013;346:f2914.

18. Moher D, Shamseer L, Clarke M, et al. Preferred reporting items for systematic review and meta-analysis protocols (PRISMA-P) 2015 statement. Syst Rev 2015;4:1.

19. Shamseer L, Moher D, Clarke M, et al. Preferred reporting items for systematic review and meta-analysis protocols (PRISMA-P) 2015: elaboration and explanation. BMJ 2015;350:g7647.

20. Higgins JPT, Altman DG, Gøtzsche PC, et al. The Cochrane collaboration's tool for assessing risk of bias in randomised trials. BMJ 2011;343:d5928.

21. Higgins JPT, Thompson SG, Deeks JJ, et al. Measuring inconsistency in meta-analyses. BMJ 2003;327:557-60.

22. Higgins JPT, Jackson D, Barrett JK, et al. Consistency and inconsistency in network meta-analysis: concepts and models for multi-arm studies. Res Synth Methods 2012;3:98-110.

23. Dias S, Welton NJ, Caldwell DM, et al. Checking consistency in mixed treatment comparison meta-analysis. Stat Med 2010;29:932-44.

24. Salanti G, Ades AE, loannidis JPA. Graphical methods and numerica summaries for presenting results from multiple-treatment metaanalysis: an overview and tutorial. J Clin Epidemiol 2011;64:163-71.

25. Chaimani A, Higgins JPT, Mavridis D, et al. Graphical tools for network meta-analysis in STATA. PLoS One 2013;8:e76654.

26. Puhan MA, Schünemann $\mathrm{HJ}$, Murad $\mathrm{MH}$, et al. A grade Working group approach for rating the quality of treatment effect estimates from network meta-analysis. BMJ 2014;349:g5630.

27. Stevens JW, Khunti K, Harvey R, et al. Preventing the progression to type 2 diabetes mellitus in adults at high risk: a systematic review and network meta-analysis of lifestyle, pharmacological and surgical interventions. Diabetes Res Clin Pract 2015;107:320-31.

28. Haw JS, Galaviz KI, Straus AN, et al. Long-term sustainability of diabetes prevention approaches: a systematic review and meta-analysis of randomized clinical trials. JAMA Intern Med 2017;177:1808-17.

29. Galaviz KI, Weber MB, Straus A, et al. Global diabetes prevention interventions: a systematic review and network meta-analysis of the real-world impact on incidence, weight, and glucose. Diabetes Care 2018;41:1526-34.

30. Holman RR, Coleman RL, Chan JCN, et al. Effects of acarbose on cardiovascular and diabetes outcomes in patients with coronary heart disease and impaired glucose tolerance (ACE): a randomised, double-blind, placebo-controlled trial. Lancet Diabetes Endocrinol 2017;5:877-86.

31. Spence JD, Viscoli CM, Inzucchi SE, et al. Pioglitazone therapy in patients with stroke and prediabetes: a post hoc analysis of the iris randomized clinical trial. JAMA Neurol 2019;76:526-35. 